\title{
Regulation of Host Factors Involved in HIV Budding Through Autoinhibition
}

\author{
Alessia Zamborlini ${ }^{1}$, Yoshiko Usami ${ }^{1}$, Sheli Radoshitzky' ${ }^{2}$, Elena Popova ${ }^{1}$, \\ Giorgio Palu ${ }^{3}$ and Heinrich Gottlinger* ${ }^{*} 1$
}

\author{
Address: ${ }^{1}$ Dept. of Molecular Medicine and Program in Gene Function and Expression, UMass Medical School, Worcester, MA, USA, ${ }^{2}$ Dana-Farber \\ Cancer Institute, Boston, MA, USA and ${ }^{3}$ Institute of Microbiology, University of Padua, Padua, Italy \\ Email: Heinrich Gottlinger* - heinrich.gottlinger@umassmed.edu \\ * Corresponding author $\ddagger$ Presenting author
}

from 2005 International Meeting of The Institute of Human Virology

Baltimore, USA, 29 August - 2 September 2005

Published: 8 December 2005

Retrovirology 2005, 2(SuppI I):SI47 doi:10.1 I86/1742-4690-2-SI-SI47

HIV-1 engages an endosomal machinery that includes the sorting complex ESCRT-III to promote virus budding. ESCRT-III components such as CHMP3 have highly acidic $\mathrm{N}$-terminal and highly basic C-terminal halfs, suggesting a model in which CHMP proteins are regulated through electrostatic interactions. Consistent with this model, we find that progressively longer truncations into the acidic domain of CHMP3 lead to an increasingly potent antiHIV budding activity, whereas the full-length molecule has no dominant-negative activity. We also find that the anti-HIV activity of CHMP3 mutants correlates strictly with their ability to interact with the isolated acidic domain in GST pulldown assays. Together, our results imply that the acidic domain of CHMP3 interacts with the $\mathrm{N}$-terminal basic domain in an autoinhibitory manner, and that the exposure of the basic domain accounts for the profound anti-HIV activity of mutant CHMP proteins. 